PROCEEDINGS OF THE AMERICAN MATHEMATICAL SOCIETY

Volume 124, Number 8, August 1996

\title{
ON THE DISTRIBUTIONS OF BOUNDARY VALUES OF CAUCHY INTEGRALS
}

\author{
ALEXEI G. POLTORATSKI
}

(Communicated by Theodore W. Gamelin)

\begin{abstract}
We use new methods to give short proofs to some known results on the distributions of boundary values of Cauchy integrals. We also indicate some further generalizations.
\end{abstract}

\section{INTRODUCTION}

Let $\varphi$ be an analytic function on the unit disk $\mathbb{D}$ such that $|\varphi| \leq 1$. Then for every $\alpha \in \mathbb{T}=\partial \mathbb{D}$ the function $(\alpha+\varphi)(\alpha-\varphi)^{-1}$ has positive real part. Therefore, there is a unique positive measure $\mu_{\alpha}$ on $\mathbb{T}$ such that

$$
\mathcal{P} \mu_{\alpha}=\operatorname{Re} \frac{\alpha+\varphi}{\alpha-\varphi}
$$

where $\mathcal{P} \mu_{\alpha}$ denotes the Poisson integral:

$$
\mathcal{P} \mu_{\alpha}=\int_{\mathbb{T}} \frac{1-|z|^{2}}{|\xi-z|^{2}} d \mu_{\alpha}(\xi) .
$$

The family $\mathcal{M}_{\varphi}=\left\{\mu_{\alpha}\right\}_{\alpha \in \mathbb{T}}$ has many interesting properties. For instance, there is the following decomposition of the normalized Lebesgue measure $m$ on $\mathbb{T}$ into the integral of the measures $\mu_{\alpha}$ (see [A1]):

$$
\int_{\mathbb{T}} \mu_{\alpha} d m(\alpha)=m \text {. }
$$

(This formula should be understood in the sense that any Lebesgue measurable set $E \subset \mathbb{T}$ is $\mu_{\alpha}$-measurable for $m$-a.e. $\alpha$ and $\int_{\mathbb{T}} \mu_{\alpha}(E) d m(\alpha)=m(E)$.)

Let now $\mu$ be a finite positive Borel measure on $\mathbb{T}$. We can always find a family $\mathcal{M}_{\varphi}$ such that $\mu \in \mathcal{M}_{\varphi}$. After that, we can use the structure of the whole family, in particular the decomposition formula (0.1), to study the boundary behavior of the Cauchy integral

$$
(\mathcal{K} \mu)(z)=\int_{\mathbb{T}} \frac{1}{1-\bar{\xi} z} d \mu(\xi),
$$

or the conjugate Poisson integral

$$
(Q \mu)(z)=2 \operatorname{Im}(\mathcal{K} \mu)(z)=\int_{\mathbb{T}} \frac{2 \operatorname{Im}(z \bar{\xi})}{|\xi-z|^{2}} d \mu(\xi)
$$

Received by the editors July 27, 1994 and, in revised form, February 24, 1995.

1991 Mathematics Subject Classification. Primary 30E20, 30D55; Secondary 42A50.

Key words and phrases. Cauchy integral, conjugate functions, singular measures.

(C)1996 American Mathematical Society 
In this note we will apply this approach to give new short proofs to some known results on the distributions of boundary values of Cauchy integrals. We will also indicate some further generalizations.

The first result in this area is probably due to G. Boole who discovered, in 1857, the following formula in the case when $\mu$ is a finite positive linear combination of point masses:

$$
m(\{Q \mu>t\})=m(\{Q \mu<-t\})=\frac{1}{\pi} \arctan \frac{\|\mu\|}{t}, \quad t>0
$$

(the functions $\mathcal{K} \mu$ and $Q \mu$ are defined almost everywhere on $\mathbb{T}$ by their nontangential boundary values).

Later on, this result was extended to the case of an arbitrary positive singular measure; see [T1], [T2], [D1]. We discuss the results of this type in Section 1.

In Section 2 we study the asymptotic behavior of $m(\{|Q \mu|>t\})$ as $t \rightarrow \infty$ for arbitrary measures. The classical result of Kolmogorov states that if $\mu \ll m$ ( $\mu$ is absolutely continuous with respect to $m$ ), then

$$
m(\{|Q \mu|>t\})<C\|\mu\| / t
$$

(the exact constant $\mathrm{C}$ was obtained by Davis in [D2]) and therefore

$$
\mu \ll m \Rightarrow m(\{|Q \mu|>t\})=o\left(\frac{1}{t}\right) .
$$

The case of an arbitrary measure was investigated by Vinogradov and Hruschev $[\mathrm{V}-\mathrm{H}]$ and Goluzina $[\mathrm{G}]$. In Section 2 we prove the Vinogradov-Hruschev result in the following refined form.

We denote by $\mathcal{M}$ the set of all finite complex Borel measures on $\mathbb{T}$.

Theorem 1. Let $\mu \in \mathcal{M}$. Then

$$
\pi t \chi_{\{|\mathcal{K} \mu|>t\}} \cdot m \underset{t \rightarrow \infty}{\stackrel{*-\text { weakly }}{\longrightarrow}}\left|\mu^{s}\right| .
$$

Moreover, if $\mu$ is a real measure, then

$$
\pi t \chi_{\{Q \mu>t\}} \cdot m \underset{t \rightarrow \infty}{\stackrel{*-\text { weakly }}{\longrightarrow}}\left|\mu^{s}\right|
$$

and

$$
\pi t \chi_{\{Q \mu<-t\}} \cdot m \underset{t \rightarrow \infty}{\stackrel{*-\text { weakly }}{\longrightarrow}}\left|\mu^{s}\right| .
$$

Here $\chi_{\{Q \mu>t\}}$ is the characteristic function of the set $\{Q \mu>t\} \subset \mathbb{T}$ and $\left|\mu^{s}\right|$ is the variation of the singular component of $\mu$.

In Section 3 we generalize (0.3) in another direction:

Theorem 2. Let $\mu, \nu \in \mathcal{M}$. Then

$$
\nu^{s} \ll \mu^{s} \Leftrightarrow \lim _{c \rightarrow \infty} \lim _{t \rightarrow \infty} t \cdot m(\{|\mathcal{K} \nu|>t\} \backslash\{|\mathcal{K} \mu|>t / c\})=0 .
$$

As was shown by Clark in $[\mathrm{C}]$, if $\varphi$ is an inner function $(|\varphi|=1$ a.e. on $\mathbb{T})$, then $\mathcal{M}_{\varphi}$ is the system of the spectral measures of all unitary one-dimensional perturbations of the model contraction with characteristic function $\varphi$. This connection with perturbation theory is even more transparent in the context of rank-one perturbations of self-adjoint operators; see [Ar], [Do], [S-W] and [S].

In some problems on rank-one perturbations it is important to understand how the resolvent function $\left\langle(A-z)^{-1} \phi, \phi\right\rangle$ of a cyclic self-adjoint operator $A$ depends on 
the choice of the vector $\phi$. In terms of Cauchy integrals this amounts to the problem of comparing the integrals $\mathcal{K} \mu$ and $\mathcal{K} \nu$ of two equivalent measures. Theorem 2 above gives a partial solution to this problem.

The author is grateful to N. Makarov for his help in the preparation of this paper and to D. Sarason for many useful comments.

\section{Metric properties of COnJugate functions.}

In addition to (0.1) the family $\mathcal{M}_{\varphi}$ has the following properties; see [A1]. If $\mu_{\alpha}^{s}$ and $\mu_{\alpha}^{a . c .}$ are the singular and absolutely continuous components of $\mu_{\alpha}$ and $\Sigma=\{\xi \in \mathbb{T}|| \varphi \mid=1\}$, then

$$
\chi_{\Sigma} \cdot m=\int_{\mathbb{T}} \mu_{\alpha}^{s} d m(\alpha)
$$

and

$$
\left(1-\chi_{\Sigma}\right) \cdot m=\int_{\mathbb{T}} \mu_{\alpha}^{a . c .} d m(\alpha) .
$$

An analogous result for the real line is contained in [S]. Instead of the measures $\mu_{\alpha}$ Simon integrates the spectral measures of one-dimensional perturbations of a self-adjoint operator.

Let $w: \mathbb{R} \rightarrow \mathbb{T}$ be the mapping

$$
w(\lambda)=\frac{\lambda+i}{\lambda-i}
$$

In this paper we will use (1.1) in the following form:

Lemma 1.1. Let $\varphi$ be an analytic function in $\mathbb{D},|\varphi| \leq 1$ and $\varphi(0) \in \mathbb{R}$. Let $\left\{\mu_{\alpha}\right\}_{\alpha \in \mathbb{T}}=\mathcal{M}_{\varphi}$ and $\Lambda=\left\{\frac{d \mu_{1}}{d m}>0\right\}$. Then for any $t>0$

$$
\chi_{\left(\left\{Q \mu_{1}>t\right\} \backslash \Lambda\right)} \cdot m=\int_{w((t ; \infty))} \mu_{\alpha}^{s} d m(\alpha) ;
$$

in particular,

$$
m\left(\left\{Q \mu_{1}>t\right\} \backslash \Lambda\right)=\int_{w((t ; \infty))}\left\|\mu_{\alpha}^{s}\right\| d m(\alpha) .
$$

Proof. For each $\alpha \in \mathbb{T}$, the measure $\mu_{\alpha}^{s}$ is concentrated on the set

$$
\Sigma_{\alpha}=\left\{\xi \mid \lim _{\substack{\not \rightarrow \xi \\ \ngtr}} \varphi=\alpha\right\} .
$$

Since the set $\Sigma$ from (1.1) coincides up to a set of Lebesgue measure 0 with the set $\mathbb{T} \backslash \Lambda$ and since

$$
Q \mu_{1}=\operatorname{Im} \frac{1+\varphi}{1-\varphi}
$$

we have that up to a set of Lebesgue measure 0

$$
\left\{Q \mu_{1}>t\right\} \backslash \Lambda=\left\{\xi \mid \lim _{z \rightarrow \xi} \varphi \in w((t ; \infty))\right\}=\bigcup_{\alpha \in w((t ; \infty))} \Sigma_{\alpha} .
$$

That means that if we multiply both sides of formula (1.1) by $\chi_{\left\{Q \mu_{1}>t\right\} \backslash \Lambda}$, we obtain (1.3). Integrating (1.3) over $\mathbb{T}$ we obtain (1.4). 
Remark. If we dropped the condition $\varphi(0) \in \mathbb{R}$ in the statement of the corollary, we would have to replace the set $w((t ; \infty))$ in the formulas $(1.2)$ and $(1.3)$ with the set $w((t+c ; \infty))$ where $c=\operatorname{Im} \frac{1+\varphi(0)}{1-\varphi(0)}$.

Now we will give short proofs to some metric properties of conjugate functions of positive measures.

We denote by $\mathcal{M}_{+}$the subset of $\mathcal{M}$ consisting of all nonnegative measures.

Theorem 1.2. Let $\mu \in \mathcal{M}_{+}, t>0$ and $\Lambda=\left\{\frac{d \mu}{d m}>0\right\}$. Then

$$
\begin{aligned}
& \frac{1}{\pi} \arctan \frac{\|\mu\|}{t}-m(\Lambda) \leq m(\{Q \mu>t\} \backslash \Lambda) \leq \frac{1}{\pi} \arctan \frac{\|\mu\|}{t}, \\
& \frac{1}{\pi} \arctan \frac{\|\mu\|}{t}-m(\Lambda) \leq m(\{Q \mu<-t\} \backslash \Lambda) \leq \frac{1}{\pi} \arctan \frac{\|\mu\|}{t} .
\end{aligned}
$$

Proof. Without loss of generality we can assume that $\|\mu\|=1$. Consider an analytic function $\varphi$ such that $\varphi(0)=0$ and

$$
\mathcal{P} \mu=\operatorname{Re} \frac{1+\varphi}{1-\varphi} .
$$

Since

$$
\left\|\mu_{\alpha}\right\|=\frac{\alpha+\varphi(0)}{\alpha-\varphi(0)}=1
$$

we have

$$
\int_{w([t ; \infty))}\left\|\mu_{\alpha}^{s}\right\| d m(\alpha) \leq m(w([t ; \infty)))=\frac{1}{\pi} \int_{t}^{\infty} \frac{d t}{1+t^{2}}=\frac{1}{\pi} \arctan \frac{1}{t} .
$$

We also have

$$
\begin{gathered}
\int_{w([t ; \infty))}\left\|\mu_{\alpha}^{s}\right\| d m(\alpha)=\int_{w([t ; \infty))} 1-\left\|\mu_{\alpha}^{a . c .}\right\| d m(\alpha) \\
=\frac{1}{\pi} \arctan \frac{1}{t}-\int_{w([t ; \infty))}\left\|\mu_{\alpha}^{a . c \cdot}\right\| d m(\alpha) \\
\geq \frac{1}{\pi} \arctan \frac{1}{t}-\int_{\mathbb{T}}\left\|\mu_{\alpha}^{a . c .}\right\| d m(\alpha)=\frac{1}{\pi} \arctan \frac{1}{t}-m(\Lambda)
\end{gathered}
$$

because

$$
\int_{\mathbb{T}}\left\|\mu_{\alpha}^{a . c .}\right\| d m(\alpha)=m(\Lambda)
$$

by (1.2). Now, if we combine (1.4), (1.5) and (1.6), we obtain (i). Formula (ii) can be proven in the same way.

Since for any two sets $A$ and $B$

$$
m(A)-m(B) \leq m(A \backslash B) \leq m(A),
$$

Theorem 1.2 implies the following result of Tsereteli: 
Corollary 1.3 ([T1], [T2]). Let $\mu \in \mathcal{M}_{+}, t>0$. Then

$$
\begin{aligned}
& \left|\frac{1}{\pi} \arctan \frac{\|\mu\|}{t}-m(\{Q \mu>t\})\right| \leq m\left(\left\{\frac{d \mu}{d m}>0\right\}\right), \\
& \left|\frac{1}{\pi} \arctan \frac{\|\mu\|}{t}-m(\{Q \mu<-t\})\right| \leq m\left(\left\{\frac{d \mu}{d m}>0\right\}\right) .
\end{aligned}
$$

In [T1] it is also shown that using this result one can prove the theorems of Riesz and Zygmund on metric properties of conjugate functions.

Both Theorem 1.2 and Corollary 1.3 imply Boole's formula (0.2). Davis in [D1] proved (0.2) using the Brownian motion.

\section{ReConstruction of the Singular PART OF A Measure}

Proof of Theorem 1. Let us first prove (ii) in three steps.

1) Let $\mu$ be a singular positive measure. Then for the measures from $\mathcal{M}_{\varphi}$, where $\mu=\mu_{1} \in \mathcal{M}_{\varphi}$ we have

$$
\mu_{\alpha} \underset{\alpha \rightarrow 1}{\stackrel{\text { weakly }}{\longrightarrow}} \mu_{1}=\mu .
$$

Indeed, the definition of $\mathcal{M}_{\varphi}$ implies that

$$
\left(\mathcal{K} \mu_{\alpha}\right)(z) \underset{\alpha \rightarrow 1}{\longrightarrow}\left(\mathcal{K} \mu_{1}\right)(z)
$$

for any $z \in \mathbb{D}$. But linear combinations of Cauchy kernels and their complex conjugates are dense in the space of continuous functions on $\mathbb{T}$.

Now, formula (1.2) gives us

$$
\pi t \chi_{\{Q \mu>t\}} \cdot m=\pi t \int_{w((t ; \infty))} \mu_{\alpha} d m(\alpha) .
$$

Since $\pi t \sim \frac{1}{m(w((t ; \infty)))}$, the right-hand side of the last equation has the same limit (if any) as

$$
\frac{1}{m(w((t ; \infty)))} \int_{w((t ; \infty))} \mu_{\alpha} d m(\alpha) .
$$

But since

$$
w(t) \underset{t \rightarrow \infty}{\longrightarrow} w(\infty)=1
$$

the expression (2.2) is just the average over the interval "tending to the point 1 ". Thus by (2.1) we obtain (ii).

2) Let $\mu$ be an arbitrary positive measure from $\mathcal{M}$. Then the relation

$$
\begin{gathered}
\left(\left\{Q \mu^{s}>(1-\epsilon) t\right\} \cup\left\{Q \mu^{a . c .}>\epsilon t\right\}\right) \\
\supset\{Q \mu>t\} \\
\supset\left(\left\{Q \mu^{s}>(1-\epsilon) t\right\} \cap\left\{Q \mu^{a . c .}>\epsilon t\right\}\right)
\end{gathered}
$$

together with part 1) and formula (0.3) implies (ii).

[This part of the proof can also be obtained from the result of Vinogradov and Hruschev (see Corollary 2.1 below) or from the results of Tsereteli, mentioned in Section 1, via the following argument, suggested by the referee. 
To prove (ii) for positive measures, let $P_{z}\left(e^{i \theta}\right)=\frac{1-|z|^{2}}{\left|e^{i \theta}-z\right|^{2}}$ be the Poisson kernel. Start with the formula

$$
\pi t \cdot m(\{Q \mu)>t\}) \underset{t \rightarrow \infty}{\longrightarrow} \mu^{s}(\mathbb{T}) .
$$

Using linear fractional transformations it follows that

$$
\pi t \cdot \int_{\{Q \mu)>t\}} P_{z} \frac{d \theta}{2 \pi} \underset{t \rightarrow \infty}{\longrightarrow} \int P_{z} d \mu^{s}
$$

for all $z$. The result then follows since linear combinations of the functions $P_{z}$ are dense in $C(\mathbb{T})$. I am thankful to the referee for this remark.]

3) Now, let $\mu=\mu_{+}-\mu_{-}$be a real measure, $\mu_{ \pm} \in \mathcal{M}_{+}, \mu_{+} \perp \mu_{-}$. Our proposition easily follows from the previous part if $\mu_{+}$and $\mu_{-}$are concentrated on closed disjoint subsets of $\mathbb{T}$, i.e., if there are closed subsets $F_{+}$and $F_{-}$of $\mathbb{T}$ such that $\left\|\mu_{ \pm}\right\|=\mu_{ \pm}\left(F_{ \pm}\right), F_{+} \cap F_{-}=\emptyset$.

If now $\mu=\mu_{+}-\mu_{-}$is an arbitrary real measure, consider disjoint closed subsets $F_{+}$and $F_{-}$of $\mathbb{T}$ such that $\mu_{ \pm}\left(F_{\mp}\right)=0, \mu_{ \pm}\left(F_{ \pm}\right)=\left\|\mu_{ \pm}\right\|-\epsilon^{2}$ where $\epsilon$ is a small positive constant. Let $\nu_{ \pm}$be the restriction of $\mu_{ \pm}$on $F_{ \pm}$. Since

$$
\{Q(\mu-\nu)>t\} \subset\left(\left\{Q\left(\mu_{+}-\nu_{+}\right)>\frac{t}{2}\right\} \cup\left\{-Q\left(\mu_{-}-\nu_{-}\right)>\frac{t}{2}\right\}\right),
$$

by $(0.2)$ we have

$$
m(\{Q(\mu-\nu)>t\}) \leq \frac{4 \epsilon^{2}}{\pi t}
$$

(because measures $\mu_{+}-\nu_{+}$and $\mu_{-}-\nu_{-}$are positive). Thus, from the relation

$$
\begin{gathered}
(\{Q \nu>(1-\epsilon) t\} \cup\{Q(\mu-\nu)>\epsilon t\}) \\
\supset\{Q \mu>t\} \\
\supset(\{Q \nu>(1-\epsilon) t\} \cap\{Q(\mu-\nu)>\epsilon t\})
\end{gathered}
$$

we obtain

$$
\pi t \chi_{\{Q \mu>t\}} \cdot m=\pi t \chi_{\{Q \nu>t\}} \cdot m+\eta,
$$

where $\eta \in \mathcal{M},\|\eta\| \leq \frac{4 \epsilon}{\pi t}\|\nu\|+\frac{4 \epsilon}{\pi t}+o\left(\frac{1}{t}\right)$. Since

$$
\pi t \chi_{\{Q \nu>t\}} \cdot m \underset{t \rightarrow \infty}{\stackrel{*-\text { weakly }}{\longrightarrow}}|\nu|
$$

we obtain (ii).

4) To prove (iii) one should replace $\mu$ in (ii) by $-\mu$.

To prove (i), let us notice that (ii) and (iii) imply (i) for real measures because for any such measure $\mu$ we have $m(\{\mathcal{P} \mu>t\})=o(1 / t)$. Let us also notice that if $\mu$ is a complex measure, then for any $\epsilon>0$ there exist real mutually singular measures $\mu_{1}, \mu_{2}, \ldots, \mu_{n}$ and real constants $\alpha_{1}, \alpha_{2}, \ldots, \alpha_{n}$ such that for $\nu=\sum_{k=1}^{n} e^{i \alpha_{n}} \mu_{n}$ we have $\|\mu-\nu\|<\epsilon$. Since the measures $\mu_{k}$ are real and mutually singular, we can prove (i) for $\nu$ using the same argument as in part 3) (we just have to deal with $\mu_{1}, \mu_{2}, \ldots, \mu_{n}$ instead of $\mu_{+}$and $\mu_{-}$). Applying (ii) and (iii) to the real and imaginary parts of $\mu-\nu$ we obtain $\pi t m(\{|\mathcal{K}(\mu-\nu)|>t\})<2 \epsilon$. Now we can finish the proof using the estimates similar to the ones we used for $\mu$ and $\mu-\nu$ from part 3 ). 
Corollary $2.1([\mathrm{~V}-\mathrm{H}])$. Let $\mu \in \mathcal{M}$. Then

$$
\pi t \cdot m(\{|\mathcal{K} \mu|>t\}) \underset{t \rightarrow \infty}{\longrightarrow}\left\|\mu^{s}\right\| .
$$

Remark. One can prove the following localized versions of (i), (ii) and (iii); cf. [G].

Let $0 \leq \theta_{1}<\theta_{2}<2 \pi, a=e^{i \theta_{1}}, b=e^{i \theta_{2}}$, and let $I \subset \mathbb{T}$ be an open arc with the ends $a$ and $b: I=\left\{e^{i \theta}: \theta_{1}<\theta<\theta_{2}\right\}$. Let $\mu \in \mathcal{M}$. Then

$$
\pi t \chi_{(\{|\mathcal{K} \mu|>t\} \cap I)} \cdot m \underset{t \rightarrow \infty}{\stackrel{*-\text { weakly }}{\longrightarrow}} \chi_{(I \cup\{a, b\})} \cdot\left|\mu^{s}\right| .
$$

If $\mu \in \mathcal{M}_{+}$, then

and

$$
\pi t \chi_{(\{Q \mu>t\} \cap I)} \cdot m \underset{t \rightarrow \infty}{\stackrel{*-\text { weakly }}{\longrightarrow}} \chi_{(I \cup\{a\})} \cdot\left|\mu^{s}\right|
$$

$$
\pi t \chi_{(\{Q \mu<-t\} \cap I)} \cdot m \underset{t \rightarrow \infty}{\stackrel{*-\text { weakly }}{\longrightarrow}} \chi_{(I \cup\{b\})} \cdot\left|\mu^{s}\right| .
$$

\section{Relations Between Cauchy integrals}

Let us consider the following corollary of Theorem 1.

Corollary 3.1 ([T2]). Let $\mu \in \mathcal{M}$. If

$$
\lim _{t \rightarrow \infty} t \cdot \min (m(\{Q \mu>t\}), m(\{-Q \mu>t\}))=0,
$$

then $\mu \ll m$.

This shows that the inverse of the statement $(0.3)$ is also true. The situation is different when we replace $m$ with an arbitrary measure from $\mathcal{M}_{+}$(see Remark after the proof of Theorem 2 below). However, we still can obtain some necessary and sufficient conditions for one measure to be absolutely continuous with respect to another measure (see Theorem 2 in the introduction).

Proof of Theorem 2. Put $\eta(c)=\left|\nu^{s}\right|-\left|c \mu^{s}\right|$. Let $\eta_{+}(c)$ and $\eta_{-}(c)$ be the positive and negative parts of $\eta(c) \quad\left(\eta(c)=\eta_{+}(c)-\eta_{-}(c), \eta_{ \pm}(c) \in \mathcal{M}_{+}, \eta_{+}(c) \perp \eta_{-}(c)\right)$.

By Theorem 1

$$
\pi t \chi_{\{|\mathcal{K} \nu|>t\}} \cdot m-\pi t \chi_{\left\{|\mathcal{K} \mu|>\frac{t}{c}\right\}} \cdot m \underset{t \rightarrow \infty}{\stackrel{*-\text { weakly }}{\longrightarrow}} \eta(c) .
$$

But

$$
\begin{gathered}
\pi t \chi_{\{|\mathcal{K} \nu|>t\}} \cdot m-\pi t \chi_{\left\{|\mathcal{K} \mu|>\frac{t}{c}\right\}} \cdot m \\
=\pi t \chi_{\{|\mathcal{K} \nu|>t\} \backslash\left\{|\mathcal{K} \mu|>\frac{t}{c}\right\}} \cdot m-\pi t \chi_{\left\{|\mathcal{K} \mu|>\frac{t}{c}\right\} \backslash\{|\mathcal{K} \nu|>t\}} \cdot m .
\end{gathered}
$$

Thus

$$
\pi t \chi_{\{|\mathcal{K} \nu|>t\} \backslash\left\{|\mathcal{K} \mu|>\frac{t}{c}\right\}} \cdot m \underset{t \rightarrow \infty}{\stackrel{*-\text { weakly }}{\longrightarrow}} \eta_{+}(c)
$$

and, in particular,

$$
\pi t m\left(\{|\mathcal{K} \nu|>t\} \backslash\left\{|\mathcal{K} \mu|>\frac{t}{c}\right\}\right) \underset{t \rightarrow \infty}{\longrightarrow}\left\|\eta_{+}(c)\right\| .
$$

It is left to notice that $\left\|\eta_{+}(c)\right\| \underset{c \rightarrow \infty}{\longrightarrow} 0$ iff $\nu^{s} \ll \mu^{s}$.

Remark. The proof shows that if measures $\mu$ and $\nu$ were real, then we could replace the sets $\{|\mathcal{K} \nu|>t\}$ and $\left\{|\mathcal{K} \mu|>\frac{t}{c}\right\}$ from the statement of the theorem with $\{Q \nu>t\}$ and $\left\{Q \mu>\frac{t}{c}\right\}$ or with $\{Q \nu<-t\}$ and $\left\{Q \mu<-\frac{t}{c}\right\}$ respectively. 
Remark. The fact that $m(\{|\mathcal{K} \nu|>t\})=o\left(\frac{1}{t}\right)$ as $t \rightarrow \infty$ for any $\nu \ll m$ can also be generalized in the following way:

Let $\mu, \nu \in \mathcal{M}, \mu>0$. Then

$$
\nu^{s} \ll \mu^{s} \Rightarrow m\left(\left\{\left|\frac{\mathcal{K} \nu}{\mathcal{K} \mu}\right|>t\right\}\right)=o\left(\frac{1}{t}\right) \quad \text { as } \quad t \rightarrow \infty .
$$

Proof. We can suppose that $\|\mu\|=1$. Theorem 2 suggests that for any $\epsilon>0$ there are positive $c$ and $T$ such that for any $t>T$

$$
m\left(\{|\mathcal{K} \nu|>t\} \backslash\left\{|\mathcal{K} \mu|>\frac{t}{c}\right\}\right)<\frac{\epsilon}{t}
$$

Thus, for $n=1,2,3, \ldots$

$$
m\left(\left\{2^{n+1} T>|\mathcal{K} \nu|>2^{n} T\right\} \backslash\left\{|\mathcal{K} \mu|>\frac{2^{n} T}{c}\right\}\right)<\frac{\epsilon}{2^{n} T} .
$$

Since $\mu \geq 0$ and $\|\mu\|=1,|\mathcal{K} \mu|>1 / 2$ on $\mathbb{D}$. Thus,

$$
\left\{\left|\frac{\mathcal{K} \nu}{\mathcal{K} \mu}\right|>t\right\} \subset\{|\mathcal{K} \nu|>t / 2\}
$$

for any $t>0$. Hence, if $n$ is big enough (such that $2^{n} T>c$ ),

$$
\left\{\left|\frac{\mathcal{K} \nu}{\mathcal{K} \mu}\right|>2^{n+1} T\right\} \subset \bigcup_{k=n}^{\infty}\left(\left\{2^{k+1} T>|\mathcal{K} \nu|>2^{k} T\right\} \backslash\left\{|\mathcal{K} \mu|>\frac{2^{k} T}{c}\right\}\right) .
$$

Hence

$$
m\left(\left\{\left|\frac{\mathcal{K} \nu}{\mathcal{K} \mu}\right|>2^{n+1} T\right\}\right) \leq \sum_{k=n}^{\infty} \frac{\epsilon}{2^{k} T} \leq \frac{\epsilon}{2^{n-1} T}
$$

and because $\epsilon$ is arbitrary we obtain (3.1).

The inverse of (3.1) is false. Even the weaker statement that $m\left(\left\{\left|\mathcal{K}_{\nu}\right|>t\right\}\right)=$ $o\left(\frac{1}{t}\right)$ implies $\operatorname{supp} \nu^{s} \subset \operatorname{supp} \mu^{s}$ is false, as is evident if

$$
\mu=\frac{1}{|\xi-1|^{\frac{1}{2}}} m, \quad \nu=\delta_{1} .
$$

We can, however, prove the following:

Let $f \in L^{1}(\mathbb{T})$. Let

$$
\Sigma_{f}=\bigcap_{t>0} \operatorname{Clos}\{|\mathcal{K} f|>t\} .
$$

Let $\mu, \nu \in \mathcal{M}, \mu=f m+\mu^{s}$. If

$$
m\left(\left\{\left|\frac{\mathcal{K} \nu}{\mathcal{K} \mu}\right|>t\right\}\right)=o\left(\frac{1}{t}\right) \text { as } t \rightarrow \infty
$$

then

$$
\operatorname{supp} \nu^{s} \subset\left(\Sigma_{f} \cup \operatorname{supp} \mu^{s}\right) .
$$


Proof. Suppose $m\left(\left\{\left|\frac{\mathcal{K} \nu}{\mathcal{K} \mu}\right|>t\right\}\right)=o\left(\frac{1}{t}\right)$ but $\left|\nu^{s}\right|\left(F_{r}\right)<\left\|\nu^{s}\right\|$ for some $r$ where $F_{r}=$ $\operatorname{Clos}\{|\mathcal{K} f|>r\} \cup \operatorname{supp} \mu^{s}$. Then there exists an open set $E$ such that $\left|\nu^{s}\right|(E)=$ $\delta>0$ and $|\mu|<T$ on $E$ for some $T>0$. But Theorem 1 implies that

$$
m(E \cap\{|\mathcal{K} \nu|>t\}) \sim \frac{\delta}{t} \quad \text { as } t \rightarrow \infty ;
$$

thus, $m\left(\left\{\left|\frac{\mathcal{K} \nu}{\mathcal{K} \mu}\right|>t\right\}\right) \geq \frac{\delta}{T \cdot t}+o\left(\frac{1}{t}\right) \neq o\left(\frac{1}{t}\right)$.

\section{REFERENCES}

[A1] A. Aleksandrov, Multiplicity of boundary values of inner functions, Izv. Acad. Nauk. Arm. SSR, Matematica 225 (1987), 490-503; English transl., Soviet J. Contemp. Math. Anal. 22 (5) (1987), no. 5, 74-87. MR 89e:30058

[A2] A. Aleksandrov, Inner functions and related spaces of pseudocontinuable functions, Proceedings of LOMI seminars 170 (1989), 7-33; English transl., J. Soviet Math. 63 (1993), 115-128. MR 91c:30063

[Ar] N. Aronszajn, On a problem of Weyl in the theory of singular Sturm-Liouville equations, Amer. J. Math. 79 (1957), 597-610. MR 19:550

[C] D. Clark, One dimensional perturbations of restricted shifts, J. Anal. Math. 25 (1972), 169-191. MR 46:692

[D1] B. Davis, On the distributions of conjugate functions of nonnegative measures, Duke Math. J. 40 (1973), 695-700. MR 48:2649

[D2] B. Davis, On the weak type $(1,1)$ inequality for conjugate functions, Proc. Amer. Math Soc. 44 (1974), 307-311. MR 50:879

[Do] W. Donoghue, On the perturbation of spectra, Comm. Pure Appl. Math. 18 (1965), 559-576. MR 32:8171

[G] M. Goluzina, On the multiplication and division of Cauchy integrals, Vestnik Leningrad. Univ. Mat. Mekh. Astronom. (1981) no. 4, 8-15; Enlgish transl., Vestnik Leningrad. Univ. Math. 14 (1982), 261-269. MR 84a:30074

[S] B. Simon, Spectral analysis of rank one perturbations and applications, Mathematical Quantum Theory. II: Schrödinger Operators (Vancouver, 1993; J. Feldman et al., editors), CRM Proc. Lecture Notes, vol. 8, Amer. Math. Soc., Providence, RI, 1995, pp. (109-149). CMP 95:12

[S-W] B. Simon and T. Wolff, Singular continuous spectrum under rank one perturbations and localisation for random Hamiltonians, Comm. Pure Appl. Math. 39 (1986), 75-90. MR $87 \mathrm{k}: 47032$

[T1] O. Tsereteli, Metric properties of conjugate functions, Chapter I in B. V. Khvedelidze, Method of Cauchy-type integrals in discontinuous boundary value problems of the theory of holomorphic functions of a complex variable, Itogi Nauki: Sovremennye Problemy Mat., vol. 7, VINITI, Moscow, 1975, pp. 18-57; English transl., J. Soviet Math. 7 (1977), 317-342. MR 56:12276

[T2] O. Tsereteli, Conjugate functions, Mat. Zametki 22 (1977), 771-783; English transl., Math. Notes 22 (1977), 921-928. MR 58:12166

[V-H] S. Vinogradov and S. Hruschev, Free interpolation in the space of uniformly converging Taylor series, Lecture Notes in Math., vol. 864, Springer-Verlag, Berlin, 1981, pp. (171213). MR 83b:30032

Department of Mathematics 253-37, California Institute of Technology, Pasadena, CALIFORNia 91125

E-mail address: aleksei@cco.caltech.edu 